\title{
NOUVELle
}

\section{Les cellules souches} ont-elles l'âge de leur niche? À la recherche d'un sérum de jouvence...

Jean-Jacques Lataillade, Philippe Brunet de la Grange, Georges Uzan, Marie-Caroline Le Bousse-Kerdilès
P. Brunet de la Grange, G. Uzan, M.C. Le Bousse-Kerdilès : Unité 972, Institut national de la santé et de la recherche médicale, Institut André Lwoff, hôpital Paul Brousse,

14, avenue Paul Vaillant Couturier,

94807 Villejuif Cedex, France.

caroline.le-bousse-kerdiles@inserm.fr

J.J. Lataillade : Département de recherche,

Centre de transfusion sanguine des armées Jean Julliard,

1 , rue lieutenant Raoul Batany,

BP 410, 92141 Clamart Cedex, France.

Unité 972, Institut national de la santé et de la recherche médicale, Institut André Lwoff, hôpital Paul Brousse,

14, avenue Paul Vaillant Couturier,

94807 Villejuif Cedex, France. ches (CS) sont localisées dans des sites anatomiques particuliers appelés «niches». Ces niches doivent avoir une réalité fonctionnelle permettant à la CS de se maintenir en quiescence et/ou de s'autorenouveler sous le contrôle de signaux cellulaires intrinsèques ou extrinsèques, humoraux et neuroendocrines. Ainsi, les niches de CS correspondent à une entité spatiotemporelle dynamique au sein de laquelle des évènements de communications intercellulaires doivent se produire au bon endroit et au bon moment, pour contrôler le destin des CS.

\section{La niche hématopoiiétique :} un sanctuaire pour cellules souches C'est R. Schofield qui, en 1978, a le premier proposé le concept d'une niche hématopoïétique, capable de réguler la survie, la quiescence, l'autorenouvellement et la différenciation des cellules souches hématopoïétiques (CSH). Cependant, ce n'est que depuis le début des années 2000 qu'un faisceau d'arguments expérimentaux concourt à la démonstration de son existence. Bien qu'encore controversées, les connaissances actuelles font état de l'existence de deux types de niches hématopoïétiques, respectivement appelées niche endostéale et niche vasculaire (Figure 1) [1]. La niche endostéale, au contact de l'os, est composée d'ostéoblastes, de fibroblastes et d'adipocytes ayant tous pour origine une cellule souche mésenchymateuse (CSM); elle comprend aussi des ostéoclastes

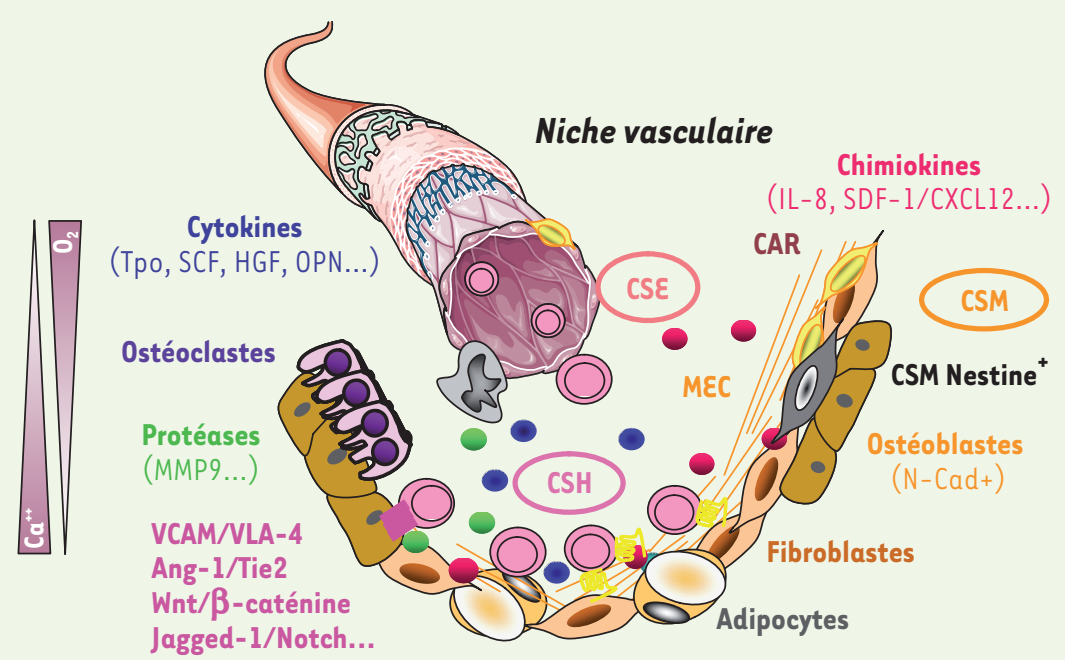

Niche endostéale impliqués dans la résorption osseuse et issus de la différenciation des CSH. La niche vasculaire, quant à elle, consiste en un réseau de vaisseaux fenêtrés formés par des cellules endothéliales provenant de cellules souches endothéliales (CSE). Les CSH entretiennent un dialogue constant avec leurs niches par l'intermédiaire de contacts directs avec différentes cellules stromales, et de facteurs diffusibles et environnementaux. Un certain nombre de couples ligands/récepteurs et de molécules d'adhérence sont impliqués dans les interactions entre ostéoblastes et CSH et réguleraient l'équilibre quiescence/ autorenouvellement de ces dernières. Les facteurs diffusibles sont, quant à eux,

Figure 1. Représentation schématique des niches hématopoïétiques médullaires. Les CSH entretiennent un dialogue constant avec leurs niches où elles sont régulées via des contacts directs avec différentes cellules stromales, ainsi que par des facteurs diffusibles et environnementaux. La niche endostéale, au contact de l'os, est majoritairement composée d'ostéoblastes et d'ostéoclastes, impliqués dans la résorption osseuse ainsi que de fibroblastes et d'adipocytes. La niche vasculaire consiste en un réseau de vaisseaux fenêtrés formés par des cellules endothéliales. CSH : cellule souche hématopoiétique; CSE : cellule souche endothéliale; CSM : cellule souche mésenchymateuse ; MEC : matrice extracellulaire; CAR : CXCL12 abondant reticular cells; $\mathrm{N}$-Cad ${ }^{+}: \mathrm{N}$-cadhérine ${ }^{+}$; MMP9 : membrane metalloproteinase 9 ; $\mathrm{Ca}$ : calcium ; 02 : oxygène. 


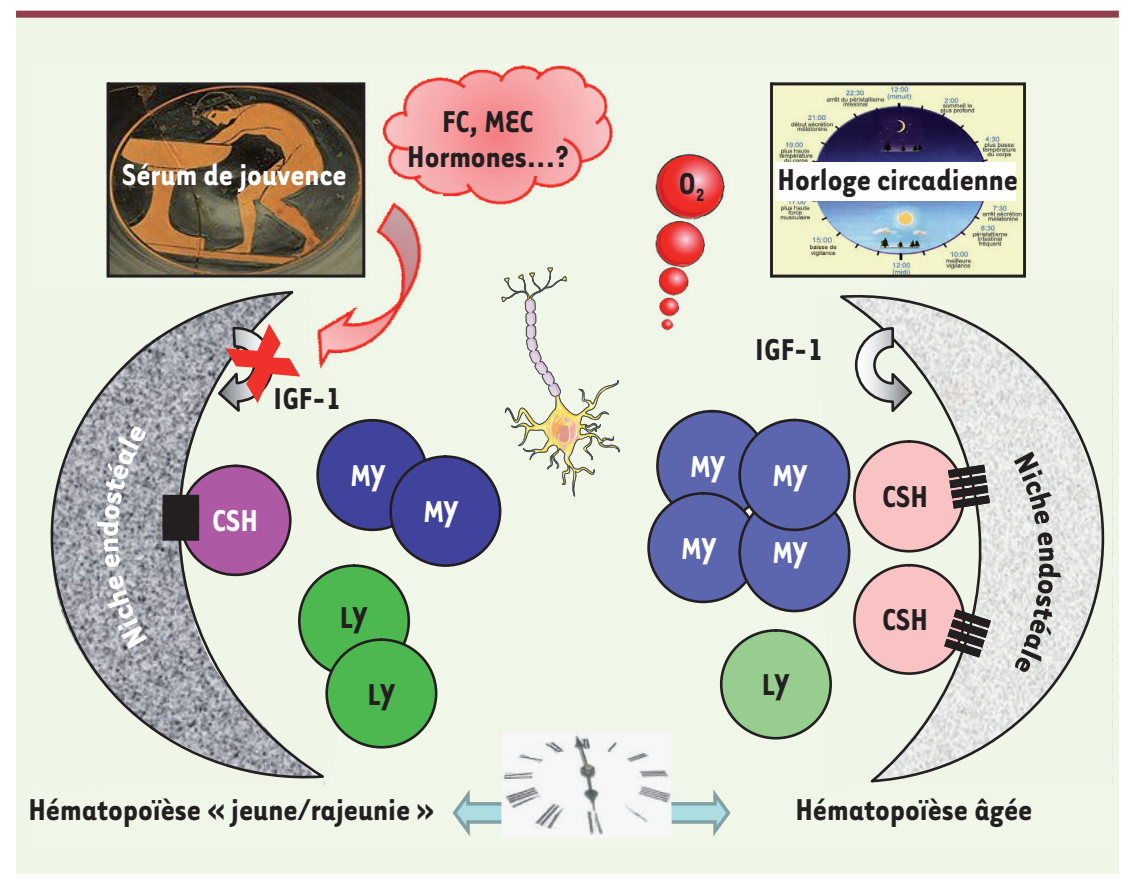

représentés par certaines hormones, des BMP (bone morphogenic protein), des cytokines et des chimiokines. Outre ces interactions cellulaires et humorales, l'importance du système nerveux sympathique dans la régulation de la prolifération et de la mobilisation des CSH a été également récemment suggérée [2].

\section{Quand l'hématopoiìse prend de l'âge}

Le vieillissement est un processus complexe qui conduit à une diminution progressive de la capacité de régénération des tissus et organes lésés. L’hématopoïèse n'échappe pas à cette règle puisque l'on observe, chez le sujet âgé, un déséquilibre entre lymphopoïèse et myélopoïèse au bénéfice des cellules myéloïdes se traduisant par une immunosénescence, une réponse émoussée aux stress hématopoïétiques et une tendance à l'anémie [3]. De façon paradoxale, malgré une augmentation du nombre total de CSH et de la fraction de celles qui sont en cycle, leurs performances diminuent en particulier leur aptitude à reconstituer les différentes lignées de cellules sanguines spécialisées dans un système expérimental de transplantation médullaire. II en est de même de leur capacité de nichage et d'adhérence, ce qui s'accompagne d'une augmentation de leur mobilisation, c'est-à-dire de leur passage de la moelle osseuse vers le sang [4]. Bien que les mécanismes classiques de sénescence cellulaire conduisent à un arrêt irréversible de la prolifération, cela ne semble pas être le cas pour les CSH. Cependant, malgré les progrès considérables réalisés dans la compréhension de la régulation de l'hématopoïèse, on ne comprend toujours pas pourquoi, ni comment, l'hématopoïèse vieillit et les CSH deviennent moins fonctionnelles avec le temps. En effet, elles répondent de façon plus faible et retardée à des stress hématologiques majeurs tels que hémorragies et infections sévères, irradiation, chimiothérapies. Les CSH sont également davantage exposées à l'émergence de mutations oncogéniques comme le montre la fréquence plus élevée d'anomalies cytogénétiques et l'incidence accrue d'hémopathies malignes chez les sujets âgés [5]. Enfin, le vieillissement du système hématopoïétique est associé à une augmentation du risque infectieux très vraisemblablement due à une diminution de la lymphopoïèse.
Figure 2. Les niches hématopoiétiques, chef d'orchestre du vieillissement de l'hématopoïèse? Des altérations de l'un ou l'autre des éléments cellulaires ou acellulaires constituant les niches hématopoïétiques sont très vraisemblablement impliquées dans le processus de vieillissement de l'hématopoïèse. Parmi ces altérations, citons les facteurs sériques (facteurs de croissance, hormones, molécules de la MEC...) qui vont agir sur la niche ostéoblastique et modifier la production locale de facteurs de croissance (FC) tels qu'IGF-1 par exemple, des variations de la teneur en oxygène et de la concentration en radicaux libres, des anomalies de régulation de l'horloge circadienne et des rythmes biologiques. CSH : cellule souche hématopoïétique; MY: cellules myéloïdes; LY: cellules lymphoïdes; IGF-1: insulin-like growth factor; FC: facteur de croissance; $M E C$ : matrice extracellulaire.

\section{L'hématopoïèse a-t-elle l'âge de} ses cellules souches ou de leur niche?

\section{Les altérations intrinsèques des CSH}

Jusqu'à présent, un certain nombre d'anomalies intrinsèques aux CSH ont été fréquemment associées au vieillissement. Parmi celles-ci, des modifications dans l'activité transcriptionnelle de gènes clés ont été proposées pour expliquer le déséquilibre lymphomyéloïde observé chez les sujets âgés. En effet, une diminution de l'expression des gènes liés à la différenciation lymphoïde et une augmentation de ceux qui sont impliqués dans la différenciation myéloïde sont observées dans les CSH. Cependant, bien que ces modifications soient essentiellement retrouvées dans les CSH et non dans les cellules matures, on ne sait pas clairement si ces altérations transcriptionnelles sont la cause ou la conséquence du processus de vieillissement [6]. Il est également intéressant de noter que les CSH des sujets âgés surexpriment le suppresseur de tumeur $\mathrm{Pl} 6^{\text {ink4a }}$, un régulateur du cycle cellulaire impliqué dans l'inhibition de la prolifération tumorale. Son expression élevée pourrait limiter la prolifération de cellules 
ayant accumulé des anomalies génétiques, mais également limiter la capacité régénératrice de ces CS [7]. De plus, malgré un relatif maintien de la longueur des télomères, une augmentation des lésions de I'ADN est fréquemment observée au cours du vieillissement. Enfin, une réduction de l'expression des gènes impliqués dans le maintien de l'intégrité génomique et du remodelage chromatinien dans ces cellules pourrait également participer au vieillissement des CSH [6].

Bien que l'ensemble de ces données suggère que les altérations de l'hématopoïèse observées au cours du vieillissement pourraient être attribuées à des anomalies intrinsèques à la CSH, les CSH sont-elles les seules responsables de cet état de fait? De telles anomalies ne résultent-elles pas plutôt de modifications de leur microenvironnement?

\section{... et les altérations de la niche}

Compte tenu du rôle crucial joué par le stroma dans la régulation des CS, il est en effet très vraisemblable qu'une altération de l'un ou l'autre des éléments cellulaires ou acellulaires constituant la niche hématopoïétique soit impliquée dans le processus de vieillissement de l'hématopoïèse. Une hypothèse similaire donnant aux niches microenvironnementales le rôle de chef d'orchestre dans le processus de vieillissement musculaire a été récemment proposée pour expliquer la sénescence des cellules satellites [8]. À ce jour, un nombre croissant d'arguments suggère qu'il en serait de même dans le système hématopoïétique. Parmi ces arguments citons les altérations de la composition cellulaire des niches médullaires comme en témoigne la perte de la masse osseuse, associée à une augmentation de l'adipogenèse et à une diminution de l'ostéogenèse, fréquemment observées chez le sujet âgé. Ces constatations sont également à rapprocher de la diminution de la fonction de support de l'hématopoïèse exercée par les cellules souches mésenchymateuses et de la longueur de leurs télomères chez les sujets âgés. Une augmentation de la sécrétion de protéines, telles que protéases et cytokines pro-inflammatoires et/ou proapoptotiques par les cellules sénescentes de la moelle vieillissante peut également conduire à une altération de l'interaction entre les CSH et leurs niches avec pour conséquence une modification de I'hématopoïèse.

II est actuellement admis que les fonctions des CSH sont étroitement dépendantes du stress oxydatif lié à la production de dérivés réactifs de l'oxygène (ROS). Ces éléments sont à prendre en compte dans la théorie mitochondriale du vieillissement où une augmentation de la production des ROS, un dysfonctionnement de la chaîne respiratoire et une accumulation de lésions de I'ADN mitochondrial participent très vraisemblablement à la pathogenèse de nombreuses maladies liées à l'âge. De façon intéressante, les CSH quiescentes sont préférentiellement localisées au contact de l'endostéum, c'est-à-dire dans les zones les plus hypoxiques de la moelle osseuse. Ainsi, chez le sujet âgé, une dérégulation du rôle protecteur de la niche endostéale sur la quiescence des CSH pourrait contribuer à l'affaiblissement de l'hématopoïèse en stimulant de façon anomale leur prolifération et en appauvrissant le nombre de celles qui sont en phase de quiescence.

Enfin, on ne peut concevoir la compréhension des mécanismes de vieillissement de l'hématopoïèse sans y intégrer l'influence de facteurs systémiques orchestrant ces différents acteurs. Une étude récente du laboratoire de P. Frenette a ainsi mis en évidence le rôle des rythmes circadiens, en lien avec une variation des concentrations médullaires de SDF-1 (stem cell derived factor-1) ou CXCL12, dans la mobilisation des CSH de la moelle vers le sang périphérique [9]. Avec le vieillissement, les rythmes biologiques s'altèrent et l'amplitude des rythmes d'expression des gènes de l'horloge biologique tend à diminuer; il ne serait donc pas surprenant que de telles altérations contribuent aux déficiences fonctionnelles de l'hématopoïèse.

Au total, compte tenu du rôle essentiel des interactions réciproques entre la $\mathrm{CSH}$ et ses niches dans la régulation de l'hématopoïèse, il apparaît difficile de dissocier les rôles respectifs des anomalies intrinsèques et extrinsèques conduisant à son vieillissement et de distinguer l'importance de l'horloge mitotique (ADN) et de l'horloge métabolique (fonction biologique) dans ce processus [10].

\section{À la recherche d'un sérum de jouvence pour un lifting des niches}

Si les niches jouent un rôle important dans le vieillissement de l'hématopoïèse, ne serait-il pas envisageable de les rajeunir pour limiter ce processus? Un tel défi a été tenté récemment par l'équipe de Mayack et al. [11], montrant, par une technique, ancienne mais très élégante, de parabiose entre des souris âgées et des souris jeunes, qu'il serait possible de rajeunir la fonction des CSH en agissant sur leur niche. Ce lifting impliquerait la présence, dans le sérum des souris jeunes, d'un ou plusieurs facteurs qui agiraient sur la niche endostéale, en particulier en réduisant la production d'IGF-1 (insulin-like growth factor-1), un facteurclé du vieillissement, par les ostéoblastes (Figure 2). Il serait donc possible d'inverser le cours du temps en transfusant les souris âgées avec du sang prélevé chez des souris jeunes ou en neutralisant la production d'IGF-l par le microenvironnement médullaire [11]. Ces résultats pourraient paraître en contradiction avec le très faible niveau d'IGF-l observé chez des centenaires, sauf si ce taux particulièrement bas est une des raisons de leur longévité ! Cependant, il est vraisemblable qu'IGF-1 régule le vieillissement de façon complexe et spécifique du tissu concerné, soulignant le rôle crucial du contexte cellulaire/microenvironnemental dans lequel il exerce ses fonctions biologiques. Ces résultats soulignent également l'importance des facteurs sériques, dont la nature est encore inconnue mais qui sont très bien conservés chez les mammifères, dans les interactions entre les CSH et leurs niches. II faut souligner cependant que, dans cette étude, les auteurs ont travaillé avec des souris $\mathrm{C} 57 \mathrm{BI} / 6$, une souche chez laquelle le nombre de CSH ainsi que le nombre de niches ostéoblastiques augmentent de façon particulièrement importante avec 
l'âge. Il serait intéressant de rechercher si le fond génétique de ces souris influence ou non le niveau d'expression de ces facteurs sériques.

Cependant, si une telle étude apporte la preuve tangible de l'existence d'un «sérum de jouvence », un grand nombre de questions restent encore sans réponse. Parmi les plus pertinentes: quelle est la nature du ou des facteurs/ molécules sériques constituant ce sérum de jouvence? Quelles en sont les cellules productrices? De quelle manière modulent-ils la concentration locale d'IGF-1 au sein des niches? Par quels mécanismes la niche endostéale ainsi «rajeunie » contrôle-t-elle le vieillissement de l'hématopoïèse? La niche vasculaire participe-t-elle à ce processus? Ces facteurs sériques jouent-ils un rôle dans les altérations de l'hématopoïèse au cours des hémopathies malignes ou dans les maladies avec une symptomatologie de vieillissement précoce?
Ainsi donc, si, comme le suggèrent les arguments cités ci-dessus, les CSH ont l'âge de leur niche, à quand leur lifting thérapeutique? Cette question reste totalement ouverte et très séduisante, cependant, elle n'en demeure pas moins ambiguë... car, en jouant ainsi avec le devenir des CSH, ne sommes-nous pas en train de revisiter la légende de Faust et d'entretenir le mythe de Dracula!! L'avenir nous le dira... peut-être ! $\diamond$ Are stem cells as old as their niches? The quest for eternal life...

\section{CONFLIT D'INTÉRÊTS}

Les auteurs déclarent n'avoir aucun conflit d'intérêts concernant les données publiées dans cet article.

\section{RÉFÉRENCES}

1. Garrett RW, Emerson SG. Bone and blood vessels: the hard and the soft of hematopoietic stem cell niches. Cell Stem Cell 2009 ; 4 : 503-6.

2. Spiegel A, Kalinkovich A, Shivtiel S, et al. Stem cell regulation via dynamic interactions of the nervous and immune systems with the microenvironment. Cell Stem Cell 2008; $3:$ 484-92.
3. Ergen AV, Goodell MA. Mechanisms of hematopoietic stem cell aging. Exp Gerontol 2010 ; 45 : 286-90.

4. Waterstrat $A$, Van Zant $G$. Effects of aging on hematopoietic stem and progenitor cells. Curr Opin Immunol 2009; 21 : 408-13.

5. Lataillade JJ, Pierre-Louis 0, Hasselbalch HC, et al. Does primary myelofibrosis involve a defective stem cell niche? From concept to evidence. Blood 2008; $112: 3026-35$.

6. Warren LA, Rossi DJ. Stem cells and aging in the hematopoietic system. Mech Ageing Dev 2009; $130: 46-53$.

7. Chambers SM, Shaw CA, Gatza C, et al. Aging hematopoietic stem cells decline in function and exhibit epigenetic dysregulation. PLoS Biol 2007 ; 5 : e201.

8. Carlson ME, Suetta C, Conboy MJ, et al. Molecular aging and rejuvenation of human muscle stem cells. EMBO Mol Med 2009 ; 1 : 381-91.

9. Méndez-Ferrer S, Lucas D, Battista M, Frenette PS Haematopoietic stem cell release is regulated by circadian oscillations. Nature 2008 ; 452 : 442-7.

10. Rando TA. Stem cells, ageing and the quest for immortality. Nature 2006 ; 441 : 1080.

11. Mayack SR, Shadrach JL, Kim FS, Wagers AJ. Systemic signals regulate ageing and rejuvenation of blood stem cell niches. Nature $2010 ; 463$ : 495-500. 